\author{
F. E. Marble \\ California Institute of Technology, \\ Karman Laboratory of Fluid Mechanics \\ and Jet Propulsion, \\ Pasadena, CA 91125
}

\section{Response of a Thin Airfoil Encountering a Strong Density Discontinuity ${ }^{1}$}

Airfoil theory for unsteady motion has been developed extensively assuming the undisturbed medium to be of uniform density, a restriction accurate for motion in the atmosphere, Glauert (1929), Burgers (1935), Theodorsen (1935), Kussner (1936), Karman and Sears (1938), Kinney and Sears (1975). In some instances, notably for airfoils comprising fan, compressor and turbine blade rows, the undisturbed medium may carry density variations or "spots," resulting from non-uniformaties in temperature or composition, of a size comparable to the blade chord. This condition exists for turbine blades, Marble (1975), Giles and Krouthen (1988), immediately downstream of the main burner of a gas turbine engine where the density fluctuations of the order of 50 percent may occur. Disturbances of a somewhat smaller magnitude arise from the ingestion of hot boundary layers into fans, Wortman (1975), and exhaust into hovercraft. Because these regions of non-uniform density convect with the moving medium, the airfoil experiences a time varying load and moment which we propose to calculate.

\section{Introduction}

If the fluid is treated as incompressible and the velocity disturbances, caused by the shape and attitude of the airfoil, are small in comparison with the uniform free stream velocity $U$, then the density field, assumed known at some time, is given as $\rho(\xi-U t, \eta)$. The complicating feature of the problem arises from the vorticity $\bar{\omega}$ generated by the interaction of the convected density field with the pressure field generated by the airfoil. For a continuous distribution of density, this vorticity satisfies the linearized relation.

$$
\left(\frac{\partial}{\partial t}+U \frac{\partial}{\partial \xi}\right) \bar{\omega}=\frac{1}{\rho^{2}} \operatorname{grad} p \times \operatorname{grad} \rho
$$

Therefore, if grad $\rho$ is large (of zeroth order), the vorticity $\bar{\omega}$ is of the same mathematical order as the pressure field of the airfoil and hence of size comparable to that distributed on the airfoil camber line. Clearly, then, solution of the thin airfoil problem involves determination of the unknown field vorticity as well as the unknown vorticity shed from the trailing edge as a result of the unsteady motion. Note that in this problem, in contrast with the conventional problem of kinematically unsteady airfoils, the time dependence arises solely from the convection of the non-uniform density fluid past the airfoil. We may note also that when the density field is of perturbation order but the pressure field is of zeroth order, a related problem arises which has been examined by Marble and Candel (1977)

\footnotetext{
'Dedicated to Professor W. R Sears on the occasion of his 80 th birthday.

Contributed by the Fluids Engineering Division for publication in the JoURNAI of Fluids Engineering. Manuscript received by the Fluids Engineering Division June 29, 193; revised manuscript received September 23, 1993. Associate Technical Editor: D. P. Telionis.
}

in a particular context, and by Goldstein (1978) under more general circumstances.

The case that will be examined in this paper is the response of a plane lifting airfoil to the passage of a strong density discontinuity normal to the direction of uniform motion of the main stream. In this circumstance a vortex sheet is formed on the density discontinuity where it is generated at a rate proportional to the pressure gradient, produced by the airfoil, along the density discontinuity. The problem will be formulated through representing the lifting plane airfoil as a sheet of vortex elements whose distribution is determined to satisfy the boundary conditions on the airfoil in the presence of $(i)$ the vortex sheet shed form the trailing edge of the airfoil and (ii) the vorticity generated in free stream by nonuniform density. Thus it will use techniques familiar from conventional formulation of thin airfoil theory but, because of the nonuniform density field, will require the introduction of some novel features.

One of these concerns the linearized field generated by a single vortex element in the presence of a strong plane density discontinuity convected by a uniform free stream of velocity $U$ parallel to the horizontal axis. A very convenient representation of this field, believed to be new, is presented which greatly simplifies determination of the vorticity distribution on the airfoil.

The determination of the distribution of wake vorticity introduces a second novel issue because, as the density jump passes over the airfoil, Kelvin's theorem may not be applied in the usual fashion. However through considering the local impulsive generation of airfoil loading, Burgers (1935), Karman and Sears (1938), it is shown that the relationship between 
airfoil circulation and shed vorticity may be indeed be represented in a form identical with that for an airfoil in a uniform density field.

\section{Vortex Element Near a Moving Density Jump}

When the fluid is uniform, an element of vorticity $\gamma\left(\xi_{1}\right)$ $d \xi_{1}$, where $\gamma\left(\xi_{1}\right)$ is the vorticity distribution on the airfoil, has a complex potential

$$
w_{0}(\zeta, t)=\frac{i \gamma\left(\xi_{1}, t\right) d \xi_{1}}{2 \pi} \ln \left(\zeta-\xi_{1}\right)
$$

where $\zeta=\xi+i \eta$ is the complex variable in the airfoil plane and $\xi_{1}$ is the coordinate of the vorticity element. When, however, the flow has a density jump from $\rho_{1}$ on the right to $\rho_{2}$ on the left, Fig. 1, the potential of the field has a discontinuity at $\xi=\lambda(t)$ but is regular on either side of this discontinuity. Therefore the actual field of the vorticity element requires the addition of a potential $w_{1}(\zeta, t)$ for $\xi>\lambda$ and a potential $w_{2}(\zeta$, $t)$ for $\xi<\lambda$. These potentials must vanish in the far field, produce equal values of $u(\lambda, \eta)$, the $\xi$-velocity component at $\xi=\lambda$, and equal values of the perturbation pressure on either side of the discontinuity, $p_{1}(\lambda, \eta)=p_{2}(\lambda, \eta)$. These pressure perturbations are related to the potentials through the Bernoulli integral,

$$
\frac{\partial \varphi_{i}}{\partial t}+U\left(\frac{\partial \varphi_{0}}{\partial \xi}+\frac{\partial \varphi_{i}}{\partial \xi}\right)=\frac{p_{i}}{\rho_{i}}
$$

and the condition to be satisfied at $\xi=\lambda(t)$ is

$$
\begin{aligned}
\rho_{1}\left(\frac{\partial \varphi_{1}}{\partial t}+U \frac{\partial \varphi_{1}}{\partial \xi}\right)-\rho_{2}\left(\frac{\partial \varphi_{2}}{\partial t}+\right. & \left.U \frac{\partial \varphi_{2}}{\partial \xi}\right) \\
& =-\left(\rho_{1}-\rho_{2}\right)\left(\frac{\partial \varphi_{0}}{\partial t}+U \frac{\partial \varphi_{0}}{\partial \xi}\right)
\end{aligned}
$$

In satisfying this condition it must be kept in mind ( $i$ ) that the potentials depend upon $\lambda(t)$ and that $d \lambda / d t=U$, and (ii) that the strength of the vorticity elements representing the airfoil will depend upon time because of the unsteady flow field.

The supplementary potentials $w_{1}$ and $w_{2}$ are analytic in the entire $\zeta$-plane although in each case only a portion of the field is used to describe the physical solution. These potentials, see Appendix, have the nature of partial images of the actual vortex element and are, in fact

$$
\begin{aligned}
& w_{1}(\zeta, t)=\frac{i \gamma\left(\xi_{1}, t\right) d \xi_{1}}{2 \pi}\left(\frac{\rho_{1}-\rho_{2}}{\rho_{1}+\rho_{2}}\right) \ln \left((\zeta-\lambda)+\left(\xi_{1}-\lambda\right)\right) \\
& w_{2}(\zeta, t)=\frac{i \gamma\left(\xi_{1}, t\right) d \xi_{1}}{2 \pi}\left(\frac{\rho_{1}-\rho_{2}}{\rho_{1}+\rho_{2}}\right) \ln \left((\zeta-\lambda)-\left(\xi_{1}-\lambda\right)\right)
\end{aligned}
$$

which hold for $\xi>\lambda$, the situation shown in Fig. 1. Specifically for $\xi<\lambda$, the complex potential $w_{0}+w_{1}$ consists of the actual vortex element plus a vortex at the image point with strength $\left(\rho_{1}-\rho_{2}\right) /\left(\rho_{1}+\rho_{2}\right)$ times that of the original. On the other hand, when $\xi_{1}<\lambda$ the complex potential $w_{0}+w_{2}$ consists of the original vortex plus a coincident vortex of strength $\left(\rho_{1}-\rho_{2}\right) /\left(\rho_{1}+\rho_{2}\right)$ times that of the original.

When the density jump has moved downstream of the vortex element, $\lambda>\xi_{1}$, the corresponding supplementary potentials are

$$
\begin{aligned}
& w_{1}(\zeta, t)=\frac{i \gamma\left(\xi_{1}, t\right) d \xi_{1}}{2 \pi}\left(\frac{\rho_{1}-\rho_{2}}{\rho_{1}+\rho_{2}}\right) \ln \left((\zeta-\lambda)-\left(\xi_{1}-\lambda\right)\right) \\
& w_{2}(\zeta, t)=\frac{i \gamma\left(\xi_{1}, t\right) d \xi_{1}}{2 \pi}\left(\frac{\rho_{1}-\rho_{2}}{\rho_{1}+\rho_{2}}\right) \ln \left((\zeta-\lambda)+\left(\xi_{1}-\lambda\right)\right)
\end{aligned}
$$

The complementary potentials, Eqs. (4), (5), allow calculation of the vorticity distribution on the discontinuity.
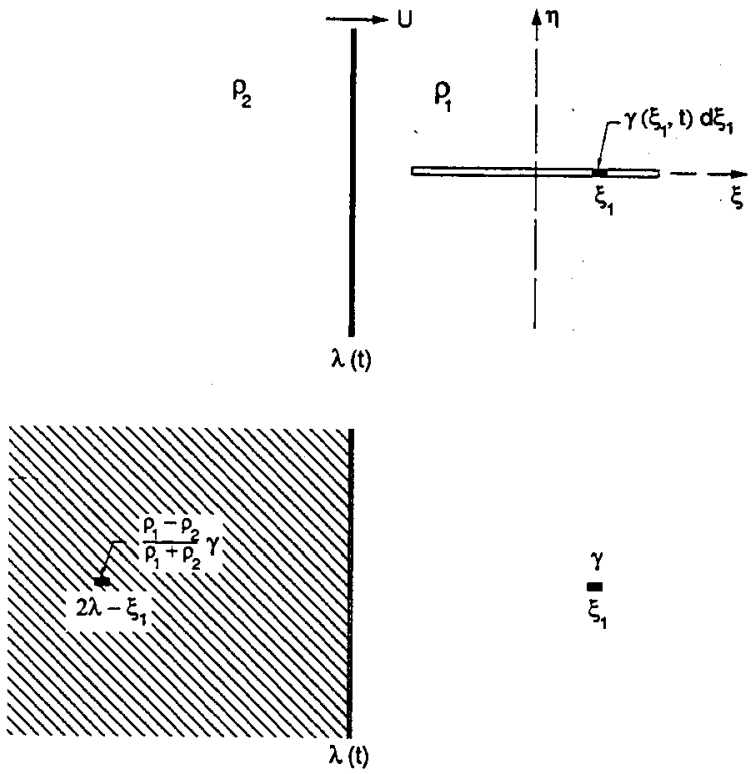

$\frac{\gamma}{\xi_{1}}$

$\lambda(t)$

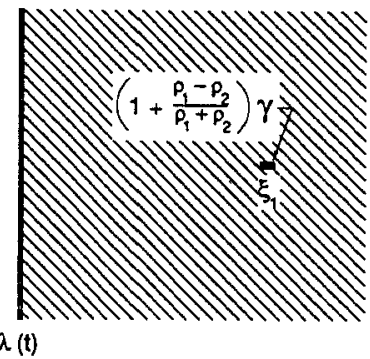

Fig. 1 Construction of flow field induced by a vortex near a convecting density jump

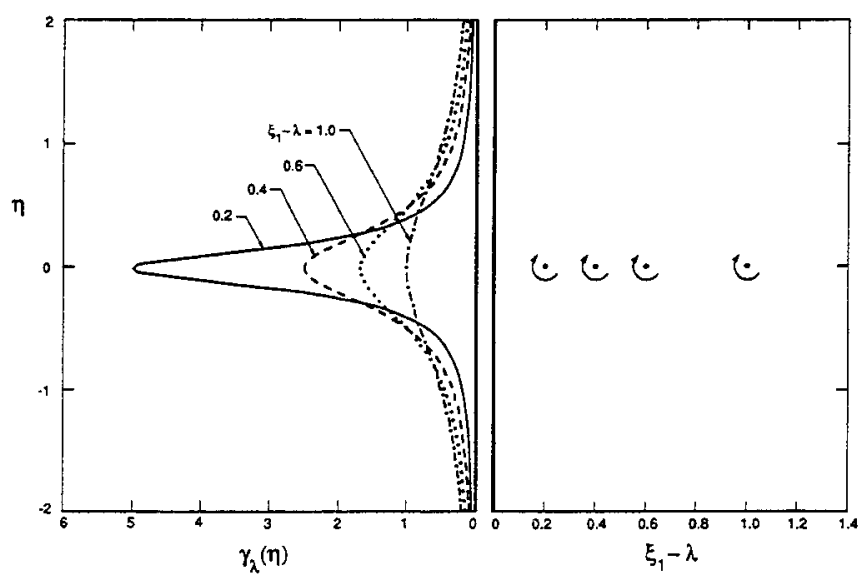

Fig. 2 Vorticity induced on density discontinuity by airfoil vortex element at various positions

$$
\gamma_{\lambda}(\eta)=-v\left(\lambda_{+}, \eta\right)+v\left(\lambda_{-}, \eta\right)=J \frac{d}{d \zeta}\left(w_{1}-w_{2}\right)_{\xi=\lambda}
$$

When the density jump is upstream of the vorticity element, $\lambda<\xi_{1}$, we find

$$
\lambda_{\lambda}(\eta)=\frac{\gamma\left(\xi_{1}, t\right) d \xi_{1}}{\pi}\left(\frac{\rho_{1}-\rho_{2}}{\rho_{1}+\rho_{2}}\right) \frac{\xi_{1}-\lambda}{\left(\xi_{1}-\lambda\right)^{2}+\eta^{2}}
$$

and this vorticity distribution is shown in Fig. 2 for several relative locations of the density jump and the vorticity element. As the density jump convects towards the vorticity element $\gamma\left(\xi_{1}, r\right)$, the vorticity distribution $\lambda_{\lambda}(\eta)$ concentrates nearer the 


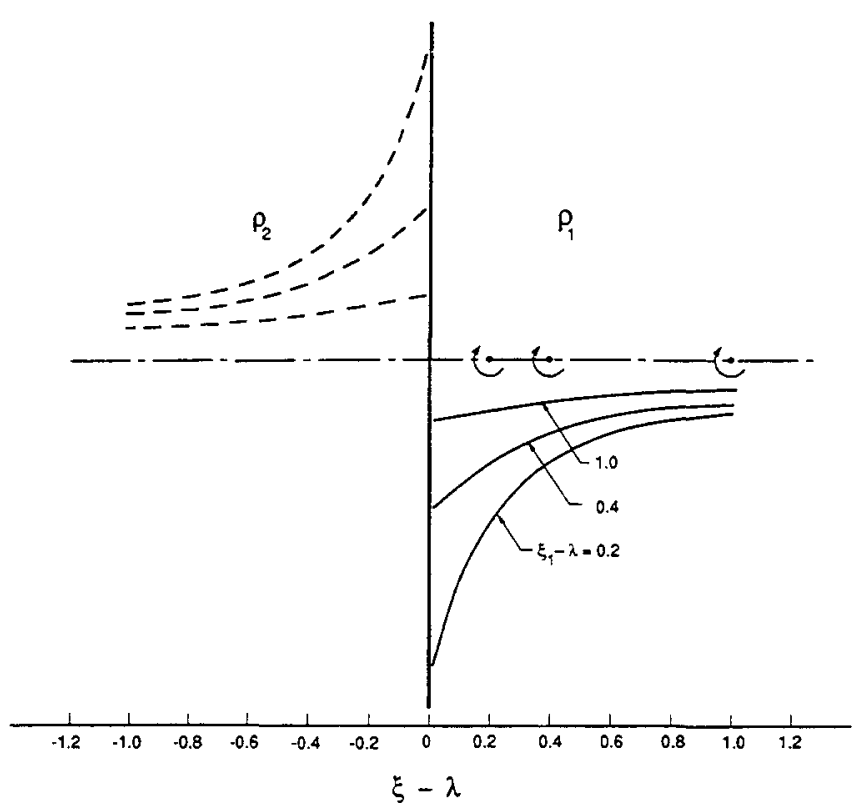

Fig. 3 Downwash induced by the vortex sheet formed on the density discontinuity

airfoil axis and has a higher maximum value. It is also easily shown, either by application of Kelvin's theorem or by direct integration of Eq. (9), that the total vorticity on the density jump is

$$
\left.\int_{-\infty}^{\infty} \gamma_{\lambda}(\eta) d \eta\right)=\frac{\gamma\left(\xi_{1}, t\right) d \xi_{1}}{2 \pi}\left(\frac{\rho_{1}-\rho_{2}}{\rho_{1}+\rho_{2}}\right)
$$

the strength of the "image vortex" we employed to construct the solution. After the density jump has passed downstream of the airfoil vorticity element, the vorticity distributions repeat themselves but with opposite sign, as might be inferred from the appropriate image vortex given by Eqs. (6), (7).

From the viewpoint of airfoil theory, the important consequence of this calculation is the downwash velocity, $v(\xi, 0)$, which is induced at the plane of the airfoil, that is $-1 \leq \xi_{1} \leq 1$. When the density jump is upstream of the airfoil vortex element, $\xi_{1}>\lambda$, the vertical velocity induced by the density jump is

$$
\begin{aligned}
& v(\xi, 0)=-\left(\frac{\rho_{1}-\rho_{2}}{\rho_{1}+\rho_{2}}\right) \frac{\gamma\left(\xi_{1}, t\right) d \xi_{1}}{2 \pi} \frac{1}{(\xi-\lambda)+\left(\xi_{1}-\lambda\right)} ; \xi>\lambda \\
& v(\xi, 0)=-\left(\frac{\rho_{1}-\rho_{2}}{\rho_{1}+\rho_{2}}\right) \frac{\gamma\left(\xi_{1}, t\right) d \xi_{1}}{2 \pi} \frac{1}{(\xi-\lambda)-\left(\xi_{1}-\lambda\right)} ; \xi<\lambda
\end{aligned}
$$

and after the density jump has passed the vorticity element, $\xi_{1}<\lambda$, the corresponding downwash velocity is

$$
\begin{aligned}
& v(\xi, 0)=\left(\frac{\rho_{1}-\rho_{2}}{\rho_{1}+\rho_{2}}\right) \frac{\gamma\left(\xi_{1}, t\right) d \xi_{1}}{2 \pi} \frac{1}{(\xi-\lambda)+\left(\xi_{1}-\lambda\right)} ; \xi<\lambda \\
& v(\xi, 0)=\left(\frac{\rho_{1}-\rho_{2}}{\rho_{1}+\rho_{2}}\right) \frac{\gamma\left(\xi_{1}, t\right) d \xi_{1}}{2 \pi} \frac{1}{(\xi-\lambda)-\left(\xi_{1}-\lambda\right)} ; \xi>\lambda
\end{aligned}
$$

The downwash velocity induced by the vortex sheet on the density jump is shown in Fig. 3 for several positions of the density jump with respect to the vortex element. As is evident for the potentials $w_{1}$ and $w_{2}$, the downwash is anti-symmetric about the density jump and has maximum absolute value at the density jump of magnitude proportional to $1 /\left|\xi_{1}-\lambda\right|$.

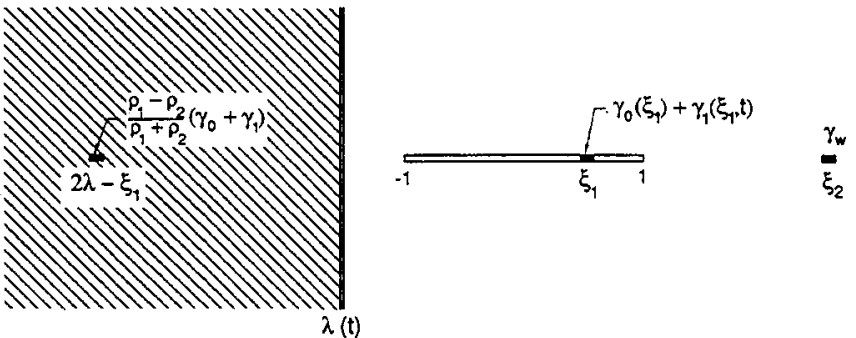

Fig. 4 Sources of downwash induction

It is important to note that the foregoing discussion pertains only to vortices that are in relative motion with respect to the density jump or whose strength varies with time. In contrast, when we consider the vortex elements that comprise the wake, the situation, as demonstrated in the Appendix, is quite different. If we follow a particular vortex element that has been shed into the wake $(i)$ it is stationary with respect to the density discontinuity, and (ii) it is of constant strength. It therefore induces a steady flow of perturbation order at the density jump and hence generates a pressure field of second order. As a consequence the vorticity on the discontinuity is influenced to only the second order and, to the present order of calculation, the potential associated with a vortex element moving in the wake has a potential continuous across the density jump.

\section{Vorticity Distribution on a Thin Airfoil}

The problem of thin airfoil theory is to determine the vorticity distribution along the camber line that provides flow tangential to the given airfoil shape $\eta_{1}(\xi)$ and regularity at the trailing edge. For steady flow of uniform density about this airfoil shape, the vorticity distribution $\gamma_{0}\left(\xi_{1}\right)$ is assumed known. For our problem, however, additional components of downwash are induced at the foil by (i) vorticity on the density discontinuity and (ii) the wake vorticity resulting from the unsteady character resulting from the convective motion of the density field. This additional downwash then necessitates a supplementary vorticity distribution $\gamma_{1}\left(\xi_{1}\right)$ on the airfoil to satisfy the boundary conditions. It is this vorticity distribution that must be found to allow pressure, force and moment on the airfoil to be calculated.

The sources of downwash are sketched in Fig. 4. At each point $\xi$ on the airfoil, the induced vertical velocity $v(\xi, 0)$ must cause the fluid to move tangentially to the airfoil surface, that is

$$
v(\xi, 0)=U \frac{d \eta_{1}}{d \xi}
$$

But the known vorticity distribution $\gamma_{0}\left(\xi_{1}\right)$ induces a flow that satisfies the condition given by Eq. (15)

$$
\frac{1}{2 \pi} \int_{-1}^{1} \frac{\gamma_{0}\left(\xi_{1}\right)}{\xi-\xi_{1}} d \xi_{1}=U \frac{d \eta_{1}}{d \xi}
$$

and consequently the remaining induction must give zero vertical velocity on the line segment $-1<\xi \leq 1$.

The remaining induction falls into three categories:

1. The direct induction of the supplementary vorticity distribution $\gamma_{1}\left(\xi_{1}\right)$ on the airfoil itself

$$
\frac{1}{2 \pi} \int_{-1}^{1} \frac{\gamma_{1}\left(\xi_{1}, t\right)}{\xi-\xi_{1}} d \xi_{1}
$$

2. The induction of the vorticity distribution on the density jump resulting from the airfoil vorticity $\gamma_{0}\left(\xi_{1}\right)+\gamma_{1}\left(\xi_{1}, t\right)$. Using the technique introduced in Section 2 , this is 


$$
\begin{aligned}
& \frac{1}{2 \pi}\left(\frac{\rho_{1}-\rho_{2}}{\rho_{1}+\rho_{2}}\right) \int_{-1}^{\lambda} \frac{\gamma_{0}+\gamma_{1}}{\xi-2 \lambda+\xi_{1}} d \xi_{1} \\
& -\frac{1}{2 \pi}\left(\frac{\rho_{1}-\rho_{2}}{\rho_{1}+\rho_{2}}\right) \int_{\lambda}^{1} \frac{\gamma_{0}+\gamma_{1}}{\xi-\xi_{1}} d \xi_{1}
\end{aligned}
$$

for values of $\xi<\lambda$, and

$$
\begin{aligned}
& \frac{1}{2 \pi}\left(\frac{\rho_{1}-\rho_{2}}{\rho_{1}+\rho_{2}}\right) \int_{-1}^{\lambda} \frac{\gamma_{0}+\gamma_{1}}{\xi-\xi_{1}} d \xi_{1} \\
& \quad-\frac{1}{2 \pi}\left(\frac{\rho_{1}-\rho_{2}}{\rho_{1}+\rho_{2}}\right) \int_{\lambda}^{1} \frac{\gamma_{0}+\gamma_{1}}{\xi-2 \lambda+\xi_{1}} d \xi_{1}
\end{aligned}
$$

for values of $\lambda>\xi$.

3. The induction of the wake vorticity

$$
\frac{1}{2 \pi} \int_{1}^{\infty} \frac{\gamma_{w}}{\xi-\xi_{2}} d \xi_{2}
$$

recalling, from the comment at the end of Section 2 , that a wake vortex element does not require an "image" in the density discontinuity.

The expressions in Eqs. (18), (19) are written explicitly for the position $\lambda(t)$ of the density jump lying between the leading and trailing edges of the airfoil. When the density jump is upstream of the leading edge of the airfoil, only the second term in each expression survives; similarly, when the density jump is downstream of the trailing edge, only the first term remains in each expression.

For points $\xi$ on the airfoil where $\lambda<\xi \leq 1$, the sum of these parts vanishes at each point $\xi$ of the range.

$$
\begin{aligned}
\int_{-1}^{1} \frac{\gamma_{1}}{\xi-\xi_{1}} d \xi_{1} & +\left(\frac{\rho_{1}-\rho_{2}}{\rho_{1}+\rho_{2}}\right)\left(\int_{-1}^{\lambda} \frac{\gamma_{1}+\gamma_{0}}{\xi-\xi_{1}} d \xi_{1}\right. \\
& \left.-\int_{\lambda}^{1} \frac{\gamma_{1}+\gamma_{0}}{\xi-2 \lambda+\xi_{1}} d \xi_{1}\right)+\int_{1}^{\infty} \frac{\gamma_{w}}{\xi-\xi_{2}} d \xi_{2}=0
\end{aligned}
$$

A corresponding integral equation, utilizing Eq. (18) rather than Eq. 19, holds at each value of $\xi$ in the range $-1<\xi<$ $\lambda$. When the wake vorticity $\gamma_{w}$ is functionally related to the supplementary vorticity $\gamma_{1}\left(\xi_{1}, t\right)$, these relations constitute an integral equation determining $\gamma_{1}$ as a function of position on the airfoil and time.

\section{Vorticity Distribution in the Wake}

The strength and distribution of the wake vorticity is determined by the vorticity $\gamma_{w}^{\prime}(\tau)$ shed at any time, $\tau$, and by the fact that, in the linearized approximation, this vorticity element is transported with free-stream velocity $U$ along the horizontal axis. Then the vorticity in the wake is just

$$
\gamma_{w}\left(t-\frac{\xi_{2}-1}{U}\right)=\gamma^{\prime}{ }_{w}(\tau)
$$

that is, the wake vorticity at a position $\xi_{2}>1$ at time $t$ is equal to that shed from the trailing edge at the earlier time

$$
\tau=t-\frac{\xi_{2}-1}{U}
$$

The usual argument to demonstrate that the shed vorticity element $\gamma_{w}^{\prime}(t) U d t$ is given by the negative of the change of total circulation about the airfoil in the same time period

$$
\gamma_{w}^{\prime}(t) U d t=-\frac{d \Gamma}{d t} d t
$$

involves the application of Kelvin's theorem to a contour encompassing the entire airfoil. And although this familiar argument may be carried out when the density jump is either upstream or downstream of the airfoil, the presence of the discontinuous potential raises complications as the density jump

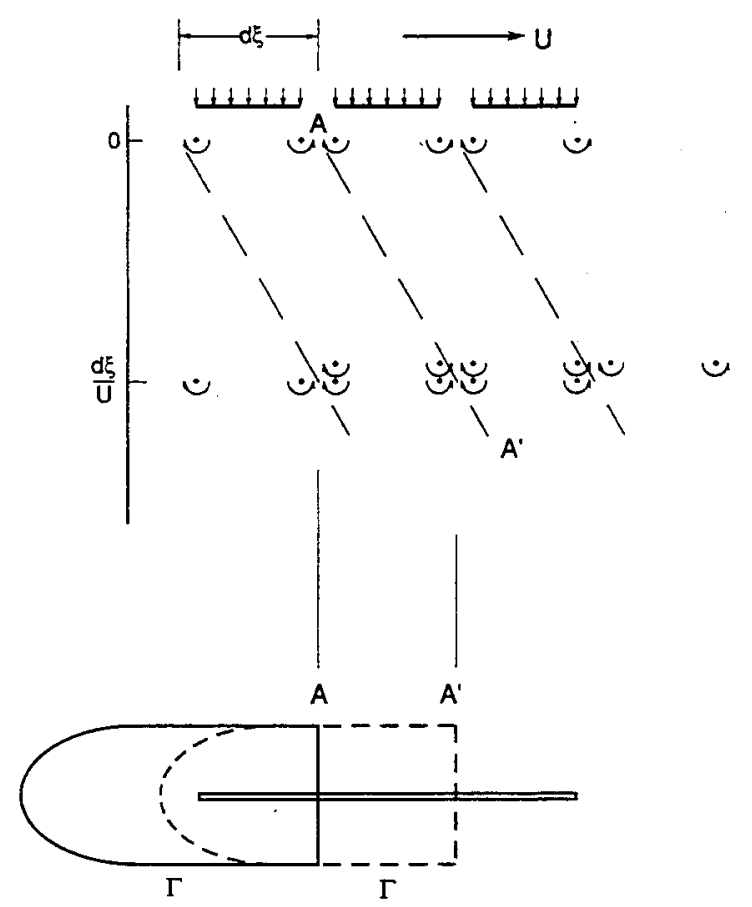

Fig. 5 Generation of lift by successive impulses-conservation of vortex pairs

passes over the airfoil. Instead, we shall employ the concept of the impulsive generation of lift described by Burgers (1935) which was used by Karman \& Sears (1938) as the basis of their unsteady airfoil theory.

An impulsive load $\delta I$ applied along an element $\delta \xi$ of the horizontal axis creates a vortex pair, each vortex having a circulation $\Gamma$, according to the relation $\delta I=\rho \Gamma \delta \xi$ where $\rho$ is density of the fluid in which impulse is applied. This vortex pair then drifts downstream with the free stream velocity $U$. A succession of impulses applied to the same element $\delta \xi$ produces a corresponding succession of vortex pairs drifting downstream along the $\xi$ axis. Now let us chose the time intervals between these impulses to be $\delta t=\delta \xi / U$ and the impulses to be of the same magnitude

$$
\delta I=\int_{0}^{\delta t} f d t
$$

$f$ being the equivalent steady load on the element. Then each impulse generates a vortex at the downstream edge of the element equal and opposite to the vortex transported from the leading edge and which was generated by the previous impulse. As a consequence there remains only the vortex of circulation $\Gamma$ at the upstream edge of the element and a vortex of strength $-\Gamma$, the one generated at the downstream edge of the element by the initial impulse, drifting far downstream. The former constitutes the "bound vortex" representing the lift on the element (more appropriately designated the "regenerate" vortex) while the latter constitutes the "starting vortex," This configuration constitute the basis for the Karman-Sears (1938) formulation of the theory of airfoils in nonuniform motion.

The complete airfoil consists of a distribution of such elements, each with its peculiar load which may vary with time, and each producing its succession of vortex pairs. Figure 5 illustrates this representation by an airfoil of three elements. At the start of the motion, $t=0$, each element produces a vortex pair according to its load and each of these vortex pairs moves downstream with the undisturbed velocity $U$. A second set of impulses is applied at a time $\delta \xi / U$ later, the resulting set of vortex pairs moves correspondingly and each successive set of impulses contributes its own set of vortex pairs. The residual 


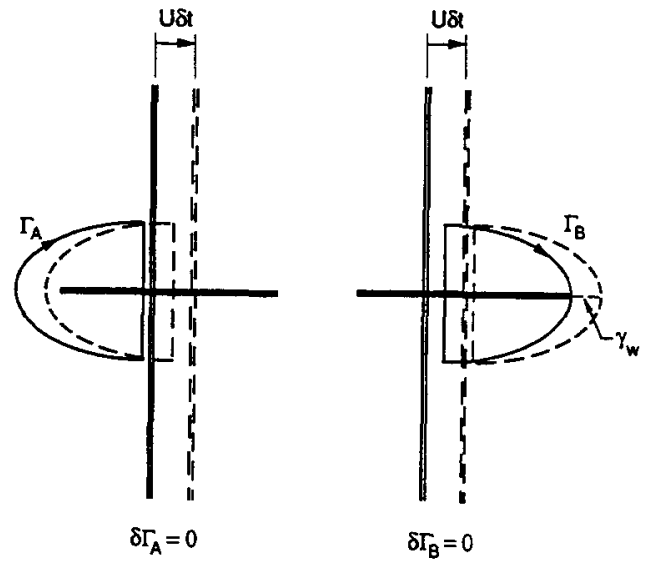

Fig. 6 Relation of shed vorticity to airfoil circulation

vortices moving away from the trailing edge constitute the vortex wake.

Now if we choose a point $A$ between two elements of the airfoil, the field to the left of this point consists of a collection of vortex pairs. Furthermore, if we move with the undisturbed fluid, this region continues to consist of vortex pairs, more being generated at each successive impulse. As a consequence, the circulation in the region does not change with time in spite of the fact that the loading of the airfoil may be changing.

This situation may be interpreted as shown also in Fig. 5 . As the contour moves with the fluid the time derivative of the circulation $\Gamma$ about this contour vanishes. Furthermore, any contour that moves with the fluid across the airfoil has a circulation that does not change, simply because any loading changes that occur create vortex pairs which remain within the contour in question.

These observations are particularly useful in determining the shed vorticity as the density discontinuity is passing over the airfoil. Referring to Fig. 6, consider the contour including the portion of the airfoil to the left of the discontinuity, lying entirely within the fluid of density $\rho_{2}$. Our argument regarding the elementary impulses holds and, as a consequence, the circulation $\Gamma_{A}$ does not change with time. On the other hand, consider the contour which contains the remaining portion of the airfoil and lying entirely within the fluid of density $\rho_{1}$. Again, as we move with the fluid, the circulation $\Gamma_{B}$ about this contour does not change. Hence as it moves downstream, the contour covers an element $U \delta t$ of the $\xi$-axis comprising a vorticity element $\gamma_{w}^{\prime}(t) U \delta t$ of the wake vorticity. Now in the time $\delta t$

$$
\delta \Gamma_{A}+\delta \Gamma_{B}=\delta \int_{-1}^{1} \gamma_{1}\left(\xi_{1}, t\right) d \xi_{1}+\gamma_{w}^{\prime}(t) U \delta t=0
$$

so that the shed wake vorticity is

$$
\gamma_{w}^{\prime}(t)=-\frac{1}{U} \frac{d}{d t} \int_{-1}^{1} \gamma_{1}(\xi, t) d \xi_{1}
$$

This result is remarkable as well as very useful! For as a consequence, we have the unanticipated advantage that the usual relationship between airfoil circulation and shed vorticity holds even as the density jump passes over the airfoil.

Equation (25), together with the relation given in Eq. (23), allows the wake vorticity distribution $\gamma_{w}\left(\xi_{1}, t\right)$ to be expressed in terms of $\gamma_{1}\left(\xi_{1}, t\right)$ so that Eq. (22) becomes an integral equation for $\gamma_{1}\left(\xi_{1}, t\right)$.

\section{Pressure Distribution, Lift and Moment}

If we denote by $u_{+}, p_{+}$and $u_{-}, p_{-}$the velocity and pressure perturbations on the upper and lower surfaces of the airfoil, the the linearization momentum equation gives

$$
\frac{\partial u}{\partial t}+U \frac{\partial u}{\partial \xi}=-\frac{1}{\rho} \frac{\partial}{\partial} \frac{p}{\xi}
$$

where the density may be $\rho_{1}$ or $\rho_{2}$ depending upon the region in which Eq. (26) is applied. Now the vorticity representing the airfoil, $\gamma(\xi, t)$, is equal to $u_{+}-u_{-}$and $p_{+} p_{-}=\Delta p$ is the airfoil load per unit area in the downward direction. By writing Eq. (26) for the upper and lower surfaces and subtracting the results we obtain the following relation between the vorticity and the airfoil loading.

$$
\frac{\partial \gamma}{\partial t}+U \frac{\partial \gamma}{\partial \xi}=-\frac{1}{\rho} \frac{\partial \Delta p}{\partial \xi}
$$

When the fluid density is uniform this may be integrated from the trailing edge, $\xi=1$, at which point $\gamma=0$ and $\Delta p=0$. Then

$$
\rho \int_{\xi}^{1} \frac{\partial \gamma}{\partial t} d \xi-\rho U_{\gamma}(\xi, t)=\Delta p(\xi, t)
$$

and when the density jump is upstream of the leading edge of the airfoil, this hold for $-1<\xi \leq 1$ and $\rho=\rho_{1}$. The lift on the airfoil is just

$$
\begin{aligned}
& L=\int_{-1}^{1}(-\Delta p) d \xi=\rho_{1} U \int_{-1}^{1} \gamma(\xi) d \xi \\
& -\rho_{1} \frac{d}{d t} \int_{-1}^{1} d \xi \int_{\xi}^{1} \gamma\left(\xi_{1}\right) d \xi_{1}=\rho_{1} U \Gamma-\rho_{1} \frac{d}{d t} \int_{-1}^{1}(1+\xi) \gamma(\xi) d \xi
\end{aligned}
$$

Likewise, after the density jump has passed downstream of the trailing edge of the airfoil, the lift is given by an expression identical with Eq. (29) with the the exception that $\rho_{1}$ is replaced by $\rho_{2}$. In each case $\gamma(\xi, t)=\gamma_{0}(\xi)+\gamma_{1}(\xi, t)$ and consequently

$$
\Gamma(t)=\int_{-1}^{1} \gamma_{0}(\xi) d \xi+\int_{-1}^{l} \gamma_{1}(\xi, t) d \xi=\Gamma_{0}+\Gamma_{1}(t)
$$

When the density jump lies between the leading and trailing edges of the airfoil, Eq. (28) holds with $\rho=\rho_{1}$ so long as $\xi>\lambda$. For the range $\lambda>\xi>-1$, Eq. 27 must be integrated from $\xi=\lambda$ to the left, which gives

$$
\rho_{2} \int_{\xi}^{\lambda} \frac{\partial \gamma}{\partial t} d \xi+\rho_{2} U(\gamma(\lambda)-\gamma(\xi))=-(\Delta p(\lambda)-\Delta p(\xi))
$$

Now the conditions at the density jump require that the pressure and the horizontal velocity components are continuous. As a consequence $\Delta p(\xi)$ and $\gamma(\xi)$ are continuous at $\xi=\lambda$ so that when we write the loading integral, Eq. (28), for the range $\lambda<\xi \leq 1$,

$$
\rho_{1} \int_{\lambda}^{1} \frac{\partial \gamma}{\partial t} d \xi-\rho_{1} U(\lambda)=\Delta p(\lambda)
$$

where the values of $\gamma(\lambda)$ and $\Delta p(\lambda)$ are identical with those occurring in Eq. (32).

Note further that because $d \lambda / d t=U$, Eq. (32) may be written

$$
\rho_{1} \int_{\lambda}^{1} \frac{\partial \gamma}{\partial t} d \xi-\rho_{1} U \gamma(\lambda)=\rho_{1} \frac{d}{d t} \int_{\lambda(t)}^{1} \gamma(\xi, t) d \xi=\Delta p(\lambda)
$$

and similarly for Eq. (31)

$$
\rho_{2} \frac{d}{d t} \int_{\xi}^{\lambda} \gamma(\xi, t) d \xi-\rho_{2} U \gamma=-\Delta p(\lambda)+\Delta p(\xi)
$$

The value of the pressure loading $\Delta p(\lambda)$ at the discontinuity may be eliminated between Eq. (33) and Eq. (34) to give the loading $\Delta p(\xi)$ in the range $-1<\xi<\lambda$ 


$$
\Delta p(\xi)=\rho_{2} U \gamma(\xi)+\rho_{1} \frac{d}{d t} \int_{\lambda}^{1} \gamma(\xi) d \xi+\rho_{2} \frac{d}{d t} \int_{\xi}^{\lambda} \gamma(\xi) d \xi
$$

Together with the relation valid for the range $\lambda<\xi<1$

$$
\Delta p(\xi)=-\rho_{1} U_{\gamma}(\xi)+\rho_{1} \frac{d}{d t} \int_{\xi}^{1} \gamma(\xi) d \xi
$$

the loading is then determined for all points of the airfoil.

The lift may now be computed, using Eqs. (35) and (36), during passage of the discontinuity over the airfoil

$$
\begin{aligned}
L=\int_{-1}^{1} & (-\Delta p) d \xi=\rho_{2} U \int_{-1}^{\lambda} \gamma(\xi) d \xi+\rho_{1} U \int_{\lambda}^{1} \gamma(\xi) d \xi \\
-\int_{-1}^{\lambda} d \xi\left\{\rho_{1} \frac{d}{d t} \int_{\lambda}^{1} \gamma\left(\xi_{1}\right) d \xi_{1}\right. & \left.+\rho_{2} \frac{d}{d t} \int_{\xi}^{\lambda} \gamma\left(\xi_{1}\right) d \xi_{1}\right\} \\
& -\int_{\lambda}^{1} d \xi\left\{\rho_{1} \frac{d}{d t} \int_{\xi}^{1} \gamma\left(\xi_{1}\right) d \xi_{1}\right\}
\end{aligned}
$$

which, after partial integration, gives

$$
\begin{aligned}
& L=\rho_{2} U \int_{-1}^{\lambda} \gamma(\xi) d \xi+\rho_{1} U \int_{\lambda}^{1} \gamma(\xi) d \xi \\
&-\rho_{2} \frac{d}{d t} \int_{-1}^{\lambda}(1+\xi) \gamma(\xi) d \xi-\rho_{1} \frac{d}{d t} \int_{\lambda}^{1}(1+\xi) \gamma(\xi) d \xi
\end{aligned}
$$

Likewise, the counter-clockwise moment about the mid-chord point is

$$
\begin{aligned}
& M=\rho_{2} U \int_{-1}^{\lambda} \gamma(\xi) \xi d \xi+\rho_{1} U \int_{\lambda}^{1} \gamma(\xi) \xi d \xi \\
& -\rho_{2} \frac{d}{d t} \int_{-1}^{\lambda} \frac{1}{2}\left(\xi^{2}-1\right) \gamma(\xi) d \xi-\rho_{1} \frac{d}{d t} \int_{\lambda}^{1} \frac{1}{2}\left(\xi^{2}-1\right) \gamma(\xi) d \xi
\end{aligned}
$$

\section{Application to the Flat Plate Airfoil}

A flat plate airfoil of chord 2 at a small angle of attack $\alpha$ has a vorticity distribution

$$
\gamma_{0}(\xi)=2 U \alpha \sqrt{\frac{1-\xi}{1+\xi}}
$$

for steady motion in a uniform medium. As a consequence the integral equation for $\gamma_{1}(\xi, t)$, Eq. (21), becomes, for $\lambda<-1$ ahead if the leading edge of the airfoil,

$$
\begin{aligned}
\int_{-1}^{1} \frac{\gamma_{1}\left(\xi_{1}, t\right)}{\xi-\xi_{1}} d \xi_{1}-\frac{\rho_{1}-\rho_{2}}{\rho_{1}+\rho_{2}} \int_{-1}^{1} \frac{\gamma_{1}\left(\xi_{1}, t\right)}{\xi-2 \lambda+\xi_{1}} d \xi_{1} \\
+\int_{1}^{\infty} \frac{\left.\gamma_{w}\left(t-\left(\xi_{2}-1\right) / U\right)\right)}{\xi-\xi_{2}} d \xi_{2}=\alpha\left(\frac{\rho_{1}-\rho_{2}}{\rho_{1}+\rho_{2}}\right) \\
\times\left(1-\sqrt{\frac{\xi-2 \lambda+1}{\xi-2 \lambda-1}}\right)
\end{aligned}
$$

where

$$
\gamma_{w}(\tau)=-\frac{1}{U} \frac{d}{d t} \int_{-1}^{1} \gamma_{1}\left(\xi_{1}, \tau\right) d \xi_{1}
$$

Corresponding expressions are easily obtained for the density jump over the airfoil, $-1<\lambda \leq 1$, and for the density jump downstream of the airfoil, $1<\lambda$. Although we may proceed analytically when either $\lambda<-1$ or $\lambda>1$, the situation is more complex when the discontinuity is passing over the airfoil and consequently $\gamma_{1}\left(\xi_{1}, t\right)$ was calculated numerically. The airfoil chord line was divided into elements, a point vortex was located

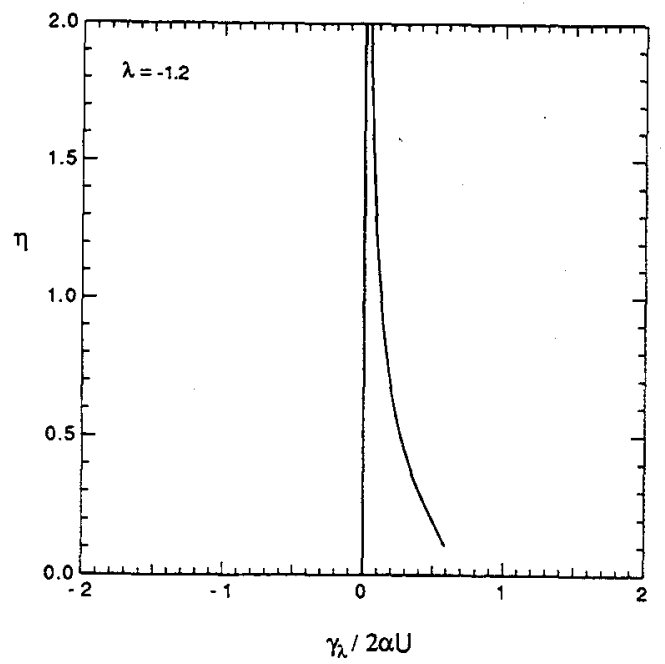

Fig. 7 (a) Vorticity on density jump

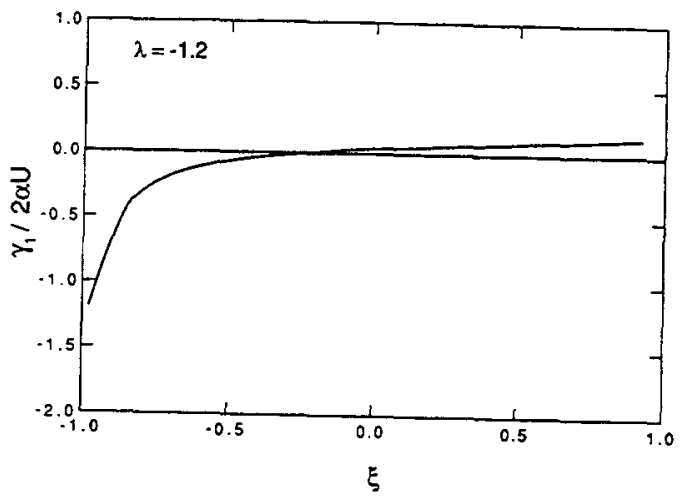

Fig. 7 (b) Vorticity on airfoil

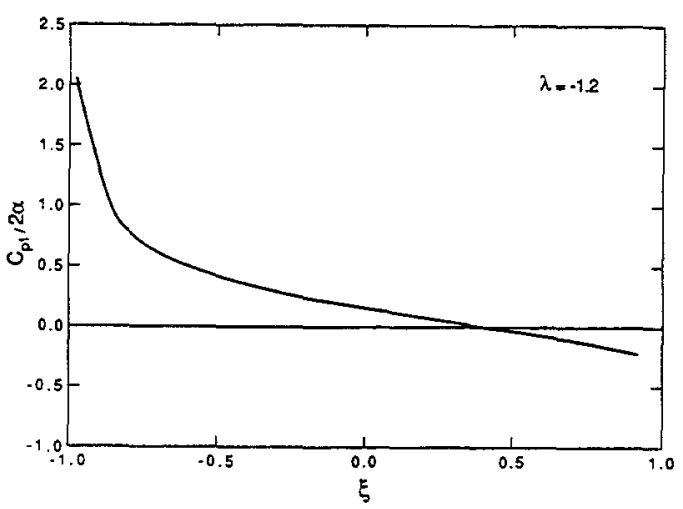

Fig. 7 (c) Pressure on airfoil

Fig. 7 Flow induced on plane airfoil by the density discontinuity, $\rho_{2} / \rho_{1}=0.5, \lambda=-1.2$

at the quarter point of each element and downwash was computed at the three-quarter point of each element. The resulting matrix was inverted to find values of $\gamma_{1}\left(\xi_{1}, t\right)$ at the discrete points of the airfoil. For most of the calculations 20 elements were used. Spot calculations using 40 points showed no substantial changes.

Examples of the vorticity distribution on the airfoil, vorticity distribution on the density jump, and pressure distribution on the airfoil are shown in Figs. 7 and 8 for values of $\lambda=-1.2$ and $\lambda=-0.8$, respectively. As the density jump approaches the leading edge of the airfoil, Fig. $7(a)$ shows that a distri- 


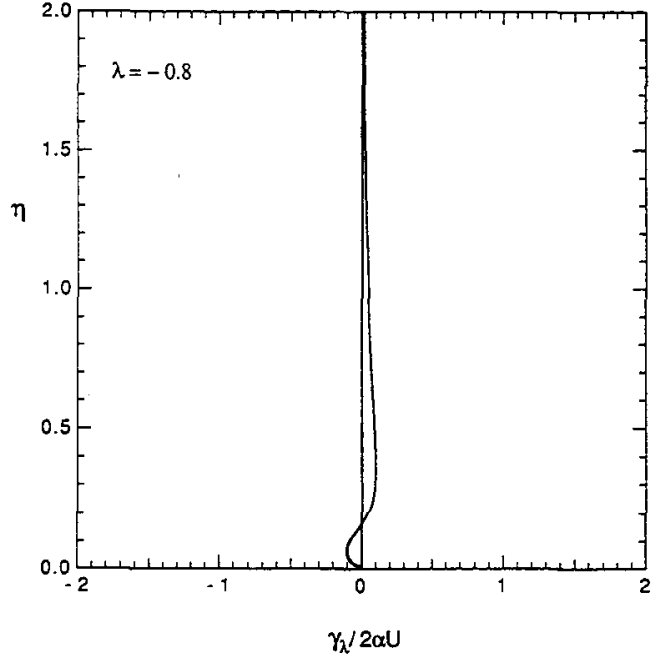

Fig. 8 (a) Vorticity on density jump

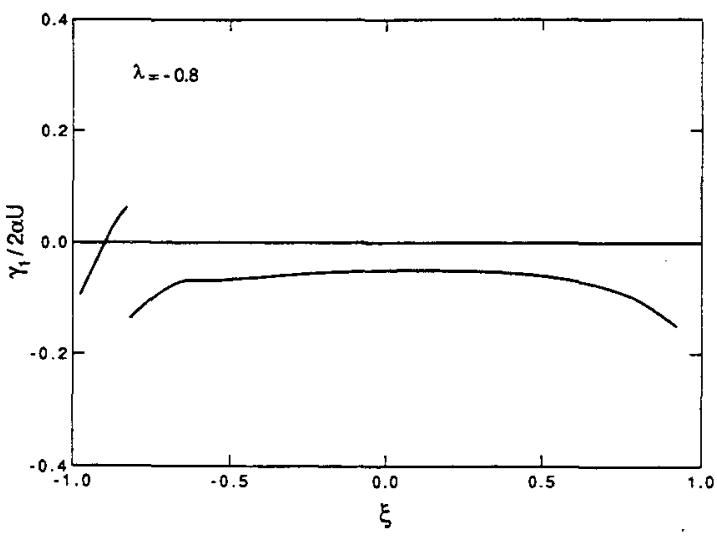

Fig. 8 (b) Vorticity on airfoil

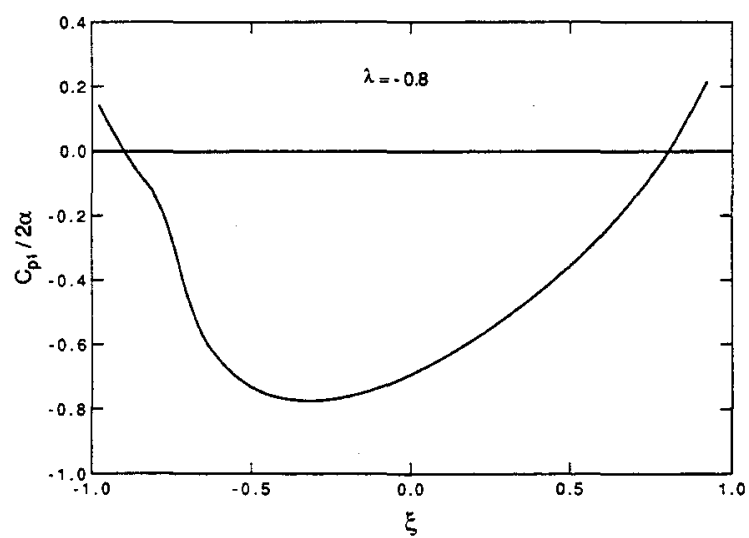

Fig. 8 (c) Pressure on airfoil

Fig. 8 Flow induced on plane airfoil by the density discontinuity, $\rho_{2} / \rho_{1}=0.5, \lambda=-0.8$

bution of positive vorticity is induced on the density jump when $\lambda=-1.2$. The sign of the distribution follows directly from Eq. (9) because the vorticity distribution $\gamma_{1}\left(\xi_{1}, t\right)$ is dominated by the initial loading $\gamma_{0}\left(\xi_{1}\right)$, Eq. (36). As a consequence the density jump induces a downwash at the leading edge producing the negative vorticity $\gamma_{1}$ show in Fig. $7(b)$. The resulting pressure coefficient perturbation (the negative of the loading), Fig. $7(c)$, shows a strong unloading of the leading edge corresponding to the action of a downward gust. It is

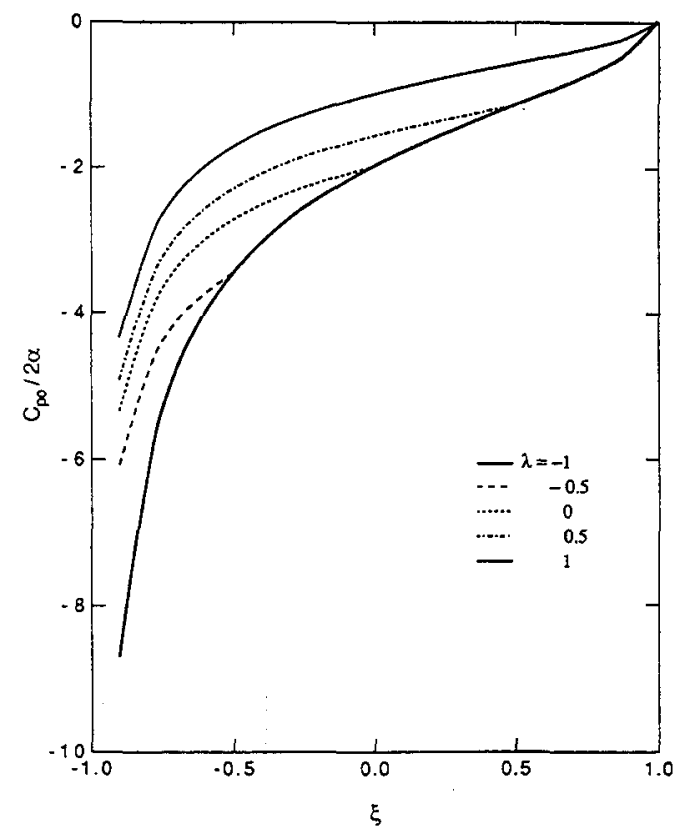

Fig. 9 Pressure distribution due to passage of density jump, initial vorticity $\gamma_{0}(\xi), \rho_{2} / \rho_{1}=0.5$

important to notice here that, in contrast to the effect of the sharp edge gust, Karman and Sears (1938), the density discontinuity has a downwash field that precedes its arrival.

The situation changes when the density jump lies within the airfoil chord, shown in Fig. 8. The vorticity distribution on the discontinuity, Fig. 8(a), shows the effect of strong leading edge vorticity $\gamma_{0}$ which now lies to the left of the density jump, largely negating the effect of the vorticity of the same sign lying to the right. Figure $8(b)$ shows a comparably small induced vorticity on the airfoil. The significant pressure coefficients in Fig. 8(c) arise largely from the local temporal variation of vorticity, the time-differentiated term of Eq. (35) and Eq. (36). The pressure distribution of Fig. 8(c) implies a significant adverse pressure gradient induced on the lower surface.

Now because the position of the airfoil and the velocity of the undisturbed flow are constant during the encounter with the density jump, the potential and the vorticity distribution $\gamma_{0}(\xi)$ given in Eq. (39) are independent of time. However, the pressure coefficient induced by this vorticity is time dependent because, specifically, the variable position $\lambda(t)$ of the discontinuity appears in the limits of the integrals of Eq. (35). When the discontinuity is ahead of the airfoil, that is $\lambda<-1$,

$$
C_{p 0} \equiv \frac{\Delta p}{1 / 2 \rho_{1} U^{2}}=-4 \alpha \sqrt{\frac{1-\xi}{1+\xi}}
$$

and after it has passed downstream, $\lambda>1$,

$$
C_{p 0}=-4 \alpha \frac{\rho_{2}}{\rho_{1}} \sqrt{\frac{1-\xi}{1+\xi}}
$$

During the process of passage, however, the pressure ahead of the discontinuity, $-1<\xi<\lambda$,

$$
C_{p 0}=-4 \alpha \frac{\rho_{2}}{\rho_{2}} \sqrt{\frac{1-\xi}{1+\xi}}-4 \alpha\left(1-\frac{\rho_{2}}{\rho_{2}}\right) \sqrt{\frac{1-\lambda}{1+\lambda}}
$$

and downstream of the discontinuity, $\lambda<\xi \leq 1$,

$$
C_{p 0}=-4 \alpha \sqrt{\frac{1-\xi}{1+\xi}}
$$




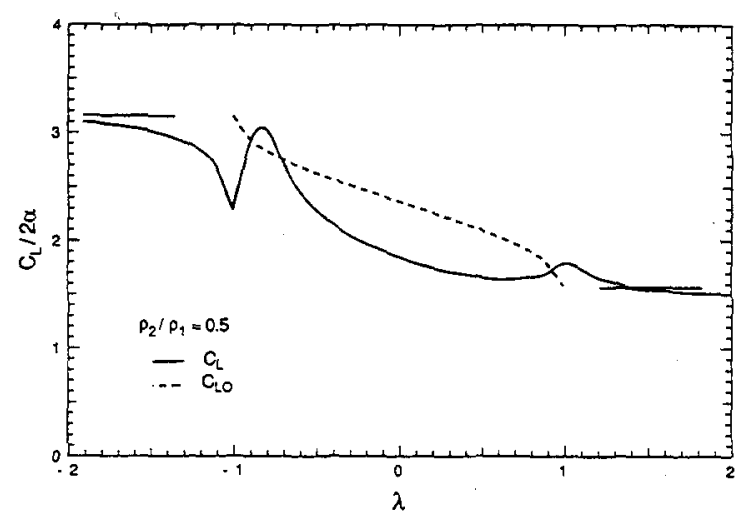

Fig. 10 Lift coefficient during passage of density discontinuity over the airloil, $\rho_{2} / \rho_{1}=0.5$

which we note is unchanged from its value when the discontinuity was far upstream of the airfoil. Figure 9 shows $C_{p 0}(\xi)$ for several values of $\lambda$ during the period the discontinuity is passing over the airfoil. The $C_{p 0}$ distribution for $\lambda=-1$ holds for all $\lambda<-1$ and the $C_{p 0}$ distribution for $\lambda=1$, equal to $\rho_{2}$ ' $\rho_{1}$ times the $C_{p 0}$ distribution for $\lambda=-1$, holds for all $\lambda>1$ after the discontinuity has left the trailing edge. The changes in the $C_{p 0}$ distribution take place from the leading edge and progress following the density jump; this feature rests upon (i) the fact that $C_{p 0}(1)=0$ and (ii) pressure continuity across the density jump. These values of $C_{p 0}$ are, generally, larger than the values of $C_{p 1}$ and we may anticipate that the forces on the airfoil will to a considerable extent be dominated by $C_{p 0}$ and thus confined to the period when the density jump is actually passing over the airfoil.

The lift coefficient $C_{L 0}$ resulting from the pressure coefficient $C_{p 0}$ is easily calculated, remembering that the airfoil chord is equal to 2 ,

$$
\begin{aligned}
C_{L 0}= & -\frac{1}{2} \int_{-1}^{1} C_{p 0} d \xi \\
& =2 \alpha\left\{\frac{\rho_{2}}{\rho_{1}} \int_{-1}^{\lambda} \sqrt{\frac{1-\xi}{1+\xi}} d \xi+\left(1+\frac{\rho_{2}}{\rho_{1}} \sqrt{\frac{1-\lambda}{1+\lambda}} \int_{-1}^{\lambda} d \xi\right.\right. \\
& \left.+\int_{\lambda}^{1} \sqrt{\frac{1-\xi}{1+\xi}} d \xi\right\}=2 \pi \alpha\left\{1-\left(1-\frac{\rho_{2}}{\rho_{1}}\right)\left(1-\frac{1}{\pi} \cos ^{-1} \lambda\right)\right.
\end{aligned}
$$

This is shown as the broken line in Fig. 10.

The clockwise moment about the airfoil midpoint is also easily calculated, again recalling that the airfoil chord is 2 ,

$$
\begin{aligned}
C_{M 0}=\frac{1}{4} \int_{-1}^{1} C_{p 0} \xi d \xi & =\frac{1}{4}(2 \alpha)\left\{\frac{\rho_{2}}{\rho_{1}} \int_{-1}^{\lambda} \sqrt{\frac{1-\xi}{1+\xi}} \xi d \xi\right. \\
& \left.+\left(1-\frac{\rho_{2}}{\rho_{1}}\right) \sqrt{\frac{1-\lambda}{1+\lambda}} \int_{-1}^{\lambda} \xi d \xi+\int_{\lambda}^{1} \sqrt{\frac{1-\xi}{1+\xi}} \xi d \xi\right\}
\end{aligned}
$$

which may be evaluated as

$$
C_{\text {Mo }}=\frac{\pi}{4} \alpha\left\{1-\left(1-\frac{\rho_{2}}{\rho_{1}}\right)\left(1-\frac{1}{\pi} \cos ^{-1} \lambda+\frac{\lambda}{\pi} \sqrt{1-\lambda^{2}}\right)\right\}
$$

This result appears as the broken line in Fig. 11 .

The complete solutions for the lift and moment coefficients shown in Figs. 10 and 11 were obtained by numerically solving Eqs. (40) and (41) for the $\gamma_{1}(\xi, t)$, the additional vorticity distribution induced by the density jump. Integration was begun with the density jump 2 chord lengths upstream, $\lambda=-4$,

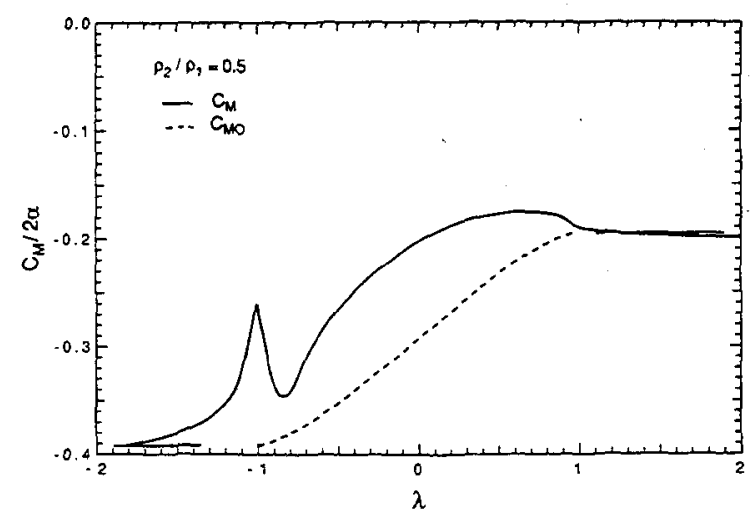

Fig. 11 Moment coefficient during passage of density discontinuity over the airfoil, $\rho_{2} / \rho_{1}=\mathbf{0 . 5}$ and continued until the density jump had passed 2 chord lengths downstream, $\lambda=4$. At $\lambda=-4$ the values of $\gamma_{1}$ were negligible and the vorticity shed from the trailing edge correspondingly small. As the discontinuity moved downstream, the successive distributions $\gamma_{1}(\xi, t)$ allowed determination of the $\gamma_{m}$ values to be used at each succeeding value of $\lambda$.

The unique features of the problem are most readily described with reference to the lift coefficients shown in Fig. 10. As the density jump approaches within about one half chord of the leading edge, the downwash, induced as illustrated in Figs. 2 and 3 , generates a reduction of the airfoil vorticity distribution, Fig. $7(b)$, and the consequent lift reduction, Fig. 9 , for $\lambda<-1$. As the discontinuity passes the leading edge, the flow following the density jump constitutes a strong upwash, creating a situation resembling the sharp-edge gust. In fact, the cusp appearing at $\lambda=-1$ is related to the lift growth for a sharp-edged gust shown in Fig. 8 of Karman and Sears (1938). The subsequent rise in $C_{L}$ overshoots the value of $C_{L 0}$ to a degree that evidently exceeds that shown simply because the number of intervals used to describe the airfoil surface was not sufficient to resolve the peak. ${ }^{2}$ The moment coefficient, Fig. 11, reflects these events in local loading. The response as the density jump passes off the trailing edge is relatively mild largely because the values of $\gamma_{0}(\xi)$ are small in this region and consequently the near-field reflected vorticity is small.

Initially it seems surprising that the variations in lift and moment are confined so closely to the period when the density discontinuity is passing directly over the airfoil, particularly so in view of our experience with other examples of unsteady airfoil behavior. Significant response to a sharp-edge gust, for example, continues until the gust has passed 5 to 10 chord lengths downstream. The difference lies in the fact that in the present case the flow field is kinematically identical when the discontinuity is far upstream and when it has passed far downstream and, in particular, the circulation about the airfoil is the same in both cases. Consequently, the shed vortex sheet contains zero net vorticity and behaves much like a vortex pair with a spacing of the order of the airfoil chord whose induced field dies out as $1 / \lambda^{2}$ rather than as $1 / \lambda$.

\section{References}

Burgers, J. M., 1935, "Problems of Non-Uniform Motion," Aerodynamic Theory, Springer, W. F. Durand, ed., pp. 280-310.

Giles, M. B., Krouthen, B., 1988, "Numerical Investigation of Hot Streaks in Turbines," Paper AIAA-88-3015, 24th Joints Propulsion Conference, Boston.

\footnotetext{
${ }^{2}$ One reviewer has pointed out that Basu and Hancock, J.F.M., Vol. 87, Pt. 1, pp. 159-178, 1978, observed a numerical anomaly resembling the behavior of the lift coefficient as the discontinuity passes the trailing edge. Although calculations of $C_{L 0}$ do not show this, the author cannot completely rule out the possibility.
} 
Glauert, H., 1929, "The Force and Moment on an Oscillating Aerofoil," British A.R.C., R.\&M. No. 1242, 1929.

Goldstein, M. E., 1978, "Unsteady Vortical and Entropic Distortions of Potential Flows Round Arbitrary Obstacles," Journal of Fluid Mechanics, Vol. 89, Part 2, pp. 433-468.

Karman, Th. v., Sears, W. R., 1938, "Airfoil Theory for Non-Uniform Motion," Jour. Aero Sci, Vol. 5, pp. 379-390.

Kinney, R. B., and Sears, W. R., 1975, "Unsteady Aerodynamics," Symposium Proceedings, Vol. I and Vol. II, University of Arizona, Tucson, R. B. Kinney, ed.

Kussner, H. G., 1936 "Zusammenfassender Bericht uber den instationaren Auftrieb von Flugeln," Luftfahrtforschung, Bd 13, pp. 410-424.

Marble, F. E. and Candel, S. M., 1977, "Acoustic Distribution from Gas Nonuniformities Convected Through a Nozzle," Journal of Sound and Vibration, Vol. 55, No. 2, pp. 225-243.

Marble, F. E., 1975, "Response of a Nozzle to an Entropy DisturbanceExample of Thermodynamically Unsteady Aerodynamics," Unsteady Aerodynamics, Vol. II, Symposium Proceedings, University of Arizona, Tucson, R. B. Kinney, ed.

Theodorsen, Th., 1935, "General Theory of Aerodynamic Instability and the Mechanism of Flutter," N.A.C.A. Technical Report No. 496, Washington, D.C.

Wortman, A., 1975, "Unsteady Flow Phenomena Causing Weapons FireAircraft Engine Inlet Interference Problems, Theory and Experiments," Unsteady Aerodynamics, V. I., Symposium Proceedings, University of Arizona, Tucson, R. B. Kinney, ed.

\section{A P P E N D I X}

Field of A Source-Vortex Near a Moving Density Discontinuity

Consider source element $\sigma(0, t) d \xi_{1}$ and a vortex element $\gamma(0, t) d \xi_{1}$ located, without loss of generality, at the origin of the $\zeta=\xi+i \eta$ plane. The complex potential is

$$
\begin{aligned}
w_{0} & =\frac{(\sigma+i \eta) d \xi_{1}}{2 \pi} \ln (\zeta) \\
& \equiv(f(t)+i g(t)) \ln (\zeta)
\end{aligned}
$$

The density discontinuity is located at $\xi=\lambda(t)$, ref Fig. 1, and moves with the undisturbed stream velocity so that $d \lambda / d t=U$. We propose to write the supplementary potentials in the form

$$
w_{1}=\left(A_{1} f+i B_{1} g\right) \ln (\zeta-2 \lambda)
$$

and

$$
w_{2}=\left(A_{2} f+i B_{2} g\right) \ln (\zeta)
$$

The potential $w_{1}$ has its singularity at the reflection point of the actual singularity and is regular to the right of the discontinuity. The potential $w_{2}$ is coincident with the actual singularity and is regular to the left of the discontinuity. The complete solutions $w_{0}+w_{1}$ and $w_{0}+w_{2}$ must satisfy the condition that the $\xi$-component of velocity be continuous at the density jump, $\xi=\lambda$, so that

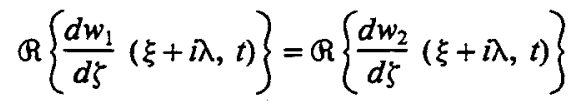

because $w_{0}$ is continuous at $\zeta=\lambda+i \eta$. The condition of Eq. A-4 is satisfied if

$$
A_{2}=-A_{1} \equiv A ; B_{2}=B_{1} \equiv B
$$

The other condition to be satisfied is that the pressure be continuous across the density jump. The perturbation pressures are given by

$$
\begin{aligned}
& p_{1} / \rho_{1}=R\left\{\left(\frac{\partial}{\partial t}+U \frac{d}{d \zeta}\right)\left(w_{0}+w_{1}\right)\right\} \\
& p_{2} / \rho_{2}=R\left\{\left(\frac{\partial}{\partial t}+U \frac{d}{d \zeta}\right)\left(w_{0}+w_{2}\right)\right\}
\end{aligned}
$$

so that pressure continuity yields the relation

$$
\begin{array}{rl}
\rho_{1} R\left\{\frac{\partial w_{1}}{\partial t}+U \frac{d w_{1}}{d \zeta}\right\}-\rho_{2} & R\left\{\frac{\partial w_{2}}{\partial t}+U \frac{d w_{2}}{d \zeta}\right\} \\
= & -\left(\rho_{1}-\rho_{2}\right) R\left\{\frac{\partial w_{0}}{\partial t}+U \frac{d w_{0}}{d \zeta}\right\}
\end{array}
$$

at $\zeta=\lambda+i \eta$. If we use the values of the potentials, Eqs. (A1), (A-2), (A-3) and the Eq. (A-5), ensuring continuity of the streamwise velocity, Eq. (A-7) may be put in the form

$$
\begin{aligned}
{\left[\left(\rho_{1}+\rho_{2}\right) A+\right.} & \left.\left(\rho_{1}-\rho_{2}\right)\right] f^{\prime} \ln \sqrt{\lambda^{2}+\eta^{2}} \\
& +\left[\left(\rho_{1}+\rho_{2}\right) B-\left(\rho_{1}-\rho_{2}\right] g^{\prime} \tan ^{-1}\left(\frac{\eta}{\lambda}\right)\right. \\
& +\left[\left(\rho_{1}+\rho_{2}\right) A+\left(\rho_{1}-\rho_{2}\right)\right] U f \frac{\lambda}{\lambda^{2}+\eta^{2}} \\
& +\left[-\left(\rho_{1}+\rho_{2}\right) B+\left(\rho_{1}-\rho_{2}\right)\right] U g \frac{\eta}{\lambda^{2}+\eta^{2}}=0
\end{aligned}
$$

For our suggested form of the solution to be valid, Eq. (A-8) must be satisfied for all values of $\eta$ and $t$. Because each function multiplying a square bracket is distinct, each of the square brackets must vanish. But these four relations consists of two identical pairs and consequently

$$
A=-\frac{\rho_{1}-\rho_{2}}{\rho_{1}+\rho_{2}}=A_{2}=-A_{1} ; \quad B=\frac{\rho_{1}-\rho_{2}}{\rho_{1}+\rho_{2}}=B_{1}=B_{2}
$$

This completes verification of the solution for the auxiliary potentials given in Eqs. (A-2) and (A-3).

We should note the special case that occurs when (i) the source and vortex strengths are independent of time, and (ii) the discontinuity is stationary with respect to the source-vortex point. This is equivalent to setting the $\partial / \partial t$ and $U$ to zero in the pressure relations, Eq. (A-6), so that the pressure disturbance vanish, to the order of our calculation, and it follows that the supplementary potentials vanish.

This circumstance arises specifically for vortices moving with the flow, such as elements of the vortex sheet shed from the trailing edge of the airfoil. These satisfy the conditions (i) and (ii) above and hence their potential is continuous through the density jump.

It is simple to generalize this result to a source element and a vortex element at an arbitrary position $\xi_{1}$ along the real axis. When the density jump is upstream of the source-vortex element, $\xi_{1}>\lambda$, the solution may be written down from the result just obtained,

$$
\begin{array}{r}
w_{0}(\zeta, t)+w_{1}(\zeta, t)=\frac{\sigma\left(\xi_{1}, t\right) d \xi_{1}}{2 \pi}\left\{\ln \left(\zeta-\xi_{1}\right)\right. \\
\left.\left.+\frac{\rho_{1}-\rho_{2}}{\rho_{1}+\rho_{2}} \ln (\zeta-\lambda)+\left(\xi_{1}-\lambda\right)\right)\right\} \\
+\frac{i \gamma\left(\xi_{1}, t\right) d \xi_{1}}{2 \pi}\left\{\ln \left(\zeta-\xi_{1}\right)+\frac{\rho_{1}-\rho_{2}}{\rho_{1}+\rho_{2}} \ln \left((\zeta-\lambda)+\left(\xi_{1}-\lambda\right)\right\}\right.
\end{array}
$$

$$
\begin{aligned}
w_{0}(\zeta, t)+ & w_{2}(\zeta, t)=\frac{\sigma\left(\xi_{1}, d \xi_{1}\right.}{2 \pi}\left\{\ln \left(\zeta-\xi_{1}\right)-\frac{\rho_{1}-\rho_{2}}{\rho_{1}+\rho_{2}} \ln \left(\zeta-\xi_{1}\right)\right\} \\
& +\frac{i \gamma\left(\xi_{1}, t\right) d \xi_{1}}{2 \pi}\left\{\ln \left(\zeta-\xi_{1}\right)+\frac{\rho_{1}-\rho_{2}}{\rho_{1}+\rho_{2}} \ln \left(\zeta-\xi_{1}\right)\right\}
\end{aligned}
$$

On the other hand, when the density discontinuity has passed downstream of the source-vortex point, this singularity now lies in the region of density $\rho_{2}$. The analytic formulation of the solution is not altered, the only modification is the interchange of indices 1 and 2 when they refer to the fluid densities 
and the regions of validity of the solutions. Therefore for $\xi_{1}$ $<\lambda$ the solutions may be written down directly

$$
\begin{aligned}
w_{0}(\zeta, t)+w_{1}(\zeta, t)= & \frac{\sigma\left(\xi_{1}, t\right) d \xi_{1}}{2 \pi}\left\{\ln \left(\zeta-\xi_{1}\right)\right. \\
& \left.+\frac{\rho_{1}-\rho_{2}}{\rho_{1}+\rho_{2}} \ln \left(\zeta-\xi_{1}\right)\right\}
\end{aligned}
$$$$
+\frac{i \gamma\left(\xi_{1}, t\right) d \xi_{1}}{2 \pi}\left\{\ln \left(\zeta-\xi_{1}\right)-\frac{\rho_{1}-\rho_{2}}{\rho_{1}+\rho_{2}} \ln \left(\zeta-\xi_{1}\right)\right\}
$$

$w_{0}(\zeta, t)+w_{2}(\zeta, t)=\frac{\sigma\left(\xi_{1}, t\right) d \xi_{1}}{2 \pi}\left\{\ln \left(\zeta-\xi_{1}\right)\right.$

$$
-\frac{\rho_{1}-\rho_{2}}{\rho_{1}+\rho_{2}} \ln \left((\zeta-\lambda)+\left(\xi_{1}-\lambda\right)\right\}
$$$$
+\frac{i \gamma\left(\xi_{1}, t\right) d \xi_{1}}{2 \pi}\left\{\ln \left(\zeta-\xi_{1}\right)-\frac{\rho_{1}-\rho_{2}}{\rho_{1}+\rho_{2}} \ln (\zeta-\lambda)+\left(\xi_{1}-\lambda\right)\right\}
$$ 